\title{
PERSEIA: a Biomedical Wireless Sensor Network to Support Healthcare Delivery for the Elderly and Chronically Ill
}

\author{
Silvia Jiménez-Fernández, Álvaro Araujo-Pinto, Antonio Cobo-Sánchez de Rojas, \\ Francisco del Pozo-Guerrero Senior Member IEEE, Octavio Nieto-Taladriz, Paula de Toledo-Heras \\ and José Manuel Moya-Fernández
}

\begin{abstract}
This paper presents a system based on Ambient Intelligence (AmI) to foster home care monitoring of senior citizens and chronically ill patients. The most important fact addressed in this research is the development of non intrusive and easy to use sensing devices. According to this, medical tests do not need user collaboration to perform them, neither powering on and off the sensor, starting the measure, configuring communications, etc.
\end{abstract}

\section{INTRODUCTION}

$\mathrm{W}$ ORLD population is aging and new technology developments need to be faced in order to support and to improve healthcare delivery, especially to those chronically ill and old. Research experiences in the past, cry out for the improvement in human-machine interfaces that enable users (i.e. mostly old people) to deal easily and nonintrusively with technology [1]-[3]. Lately, innovation in the area has come with Ambient Intelligence (AmI) [4],[5] as limitations brought on by aging are expected to be overcome by new technologies, based on the design of easy to use and to learn devices that work transparently and pervasively to aid the user [6].

Therefore, next generation follow-up and homecare systems have several important challenges to face. On the one hand, from the users' side, there is a need to develop non-intrusive systems that require a minimum humanmachine interaction and provide the right medical services on a highly-usable basis, enhancing the augmented environment while making computers disappear in the background. On the other hand, the big issue is to implement these necessities by means of affordable solutions for public and private healthcare institutions, only achievable by means of low cost structures easy to install and to maintain.

This work was supported in part by the spanish Comunidad de Madrid under Grant GR/SAL/0277/2004.

Silvia Jiménez-Fernández, Antonio Cobo-Sánchez de Rojas, Francisco del Pozo-Guerrero and Paula de Toledo-Heras are with the Bioengineering and Telemedicine Research Centre of the Technical University of Madrid Spain (\{sjimenez, acobo, fpozo, paula\}@gbt.tfo.upm.es).

Silvia Jiménez-Fernández is now Associate Professor in University of Alcalá - Spain, while Paula de Toledo-Heras is now Visiting Professor in Carlos III University of Madrid - Spain.

Álvaro Araujo-Pinto, Octavio Nieto-Taladriz, and José Manuel MoyaFernández are with the Integrated Circuits Laboratory, Technical University of Madrid - Spain (\{araujo, nieto, josem\}@die.upm.es)

\section{METHODOLOGY}

In order to achieve these requirements, a system based on Ambient Intelligence has been designed and implemented. It features a gateway, several biomedical sensors that the doctor recommends to the patient to control his/her health (pulse oximeter, electrocardiograph and accelerometer to quantify daily activity) and a user interface, essential to give commands to the user (such as start of the exam, low battery supply, etc). All network nodes are provided of wireless capabilities to allow full mobility within the Personal Area Network (PAN) [7]. The abovementioned architecture can be found in figure 1 .

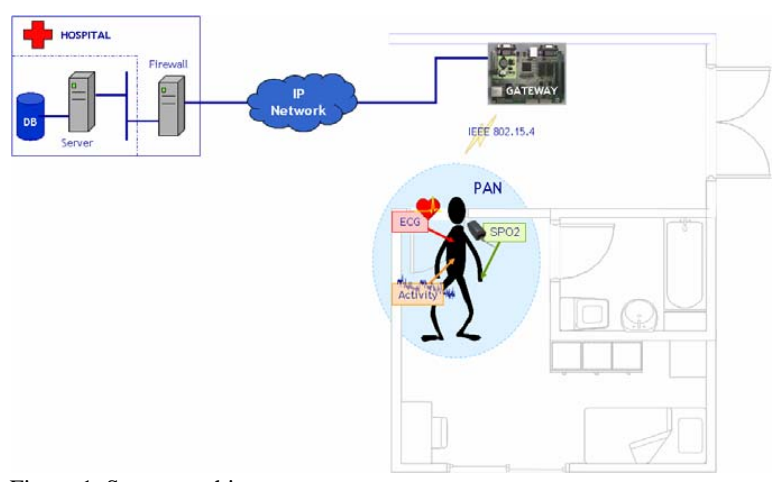

Figure 1. System architecture

\section{A. Requirements}

The requirements taken into account when designing the wireless sensor network, or personal area network, make it different to other networks, such as Internet. These are stated below:

- Self-management. The wireless network has to, automatically, route data packets using neighbor nodes as well as to adapt its topology to network changes. That is to say that the user does not have to take care of adding new nodes/sensors to the network or consider nodes' breakup.

- Context awareness. In AmI scenarios, the networks should adapt their behavior accordingly to environmental variables that govern the system: such as the actor of the system and his/her preferences, moment of the day when usage takes place, season, etc. 
- Heterogeneity and dynamism. Devices of different characteristics and nature must be interoperable and able to adapt to a network that can have different nodes depending on the environment, the user, and the application.

- Low cost. Perfectly designed networks cannot be deployed massively if they are costly. Moreover, healthcare providers will not use them in clinical routine if they are not cost-efficient or do not have important benefits over traditional healthcare delivery.

- Limited resources. Limited processing power, tight memory sizes, or low power consumption are crucial for this design. Batteries are required to last long enough to allow several measures to take place, as well as to support the wireless radio link power consumption.

- Security. Refusing intrusions, avoiding denial of service, driving the system into a safe state in case of failure, etc. are a must for real scenario setups, as sensitive and personal data will be in transit through the network.

\section{B. Functionality}

Apart from the requirements stated above, essential functionality of the different elements follows. The gateway has twofold capability: 1) it is used to communicate with the sensors that the patient has, and 2) it is needed to send data to and from the healthcare institutions (i.e. hospital, primary care, etc.). The information regarding the doctor's prescription of medical exams is sent to the gateway, where it is stored, processed and routed to the specific sensor, stating the duration, sample rate, quality of the measure, amplification factor, or any precise parameter needed to collect the data.

The sensors have been developed as stand alone wireless devices and have been provided of automatic start of the measure, auto-discovery, and auto-configuration facilities. That is to say that the patient does not need to take care of configuring nor connecting them, for instance, to upload or download the data collected. It is important to note that the user does not need to ensure either that the sensors are powered on and off, as they are suspended and resume automatically after a given time. When resume is done, every half minute, several tasks take place:

1) Checking the state of the sensor to become aware of the patient's willingness to start an exam. When the user wants to commence it, he/she places the sensor on, so that the device is able to collect data. This situation takes place no matter if the doctor programmed the test or if the patient decided to take it by himself/herself (because not feeling alright).

2) Connecting to the gateway. The radiofrequency module is powered on and a "keep alive" message is sent to the gateway. If there is information that needs to be transmitted to the device, as imminent exams that need to take place, it is sent at this moment. Afterwards, if data are still stored in the sensor, they are forwarded to the gateway.

3) Checking battery level. When batteries are close to be used up, an alarm is sent to the gateway and the user is notified so that he/she can recharge them. This check is performed every ten minutes instead of every half minute.

Once all these tasks have been performed, the device is suspended again to save batteries. Using this method, battery consumption is decreased up to a maximum of 60 percent.

\section{RESULTS}

The abovementioned approach has led to the definition and development of a system employing the concepts of Ambient Intelligence to foster independent living of the elderly.

This has been achieved using a gateway to route information from the house to the outside world (hospitals, private institutions, etc.), and also within the home environment, through a network of monitoring sensors. In order to minimize maintenance and installation costs, as well as to improve usability, a wireless network (LR-WPAN) has been used to interconnect the sensors and the hub.

Each node (i.e. sensing device) has three different parts. First, the biomedical sensor itself: an off-the-shelf OEM board (ECG, pulse oximeter) or an ad-hoc module (accelerometer) that has been provided of a second component, an autonomous power supply. As the power requirements of each sensor are different, customized supply stages have been designed using a standard interface with commercial AAA batteries, and always looking for the highest efficiency that maximizes sensors' life time. Finally, the third part that has been added provides the "intelligence" explained in the requirements section: awareness of patient's willingness to perform a measurement, network functionality (e.g. routing, reconfiguration), etc. The main component of this stage is a radio transceiver in the ISM band of 2,4 GHz that implements the physical layer and the MAC layer of the IEEE 802.15.4 wireless standard. The main characteristics featured are:

- Possible bit rates: $250 \mathrm{Kbps}, 40 \mathrm{Kbps}$ or $20 \mathrm{Kbps}$. We are using 250Kbps.

- Possible network topologies: star or point to point. We are using star topology.

- Support for low latency devices.

- Channel access using CSMA-CD (Carrier Sense Multiple Access/Collision Detection).

- Dynamic address allocation.

- Low power consumption.

Therefore, our network requirements and functionality suit perfectly with the given standard. In order to implement 
the remaining layers of the protocol stack a low-cost PIC $16 \mathrm{~F}$ microcontroller that connects to the radio transceiver through a SPI communication interface has been used. The commonly used OSI architecture has been refused in favor of an architecture based on services. The goal pursued with this design is the implementation of applications that arise as a mere aggregation of services and relations between services, rather than a node-focused orientation, simplifying the development of user applications [8]. Our services are built on top of a communication middleware [9], [10] that abstracts network or hardware specific tasks to the application. This middleware allows sending data to any node of the network without considering its location, sending broadcast messages, etc, and all this happens using a simple and unique interface (based on query-response) that can be dynamically instantiated in any node at any time. This allows to easily compose applications taking into account many complex aspects, such as privacy, safety, load balancing, low energy consumption, etc [11], [12], and in a totally transparent way to the user. The protocol stack can be depicted in figure 2 .

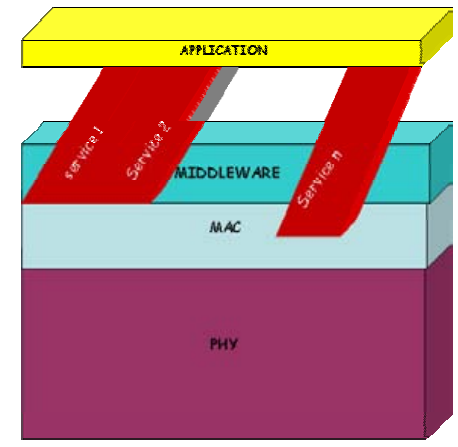

Figure 2. Protocol stack

The idea behind all this approach is to isolate common behavior that can be reused from other applications and to encapsulate it as system services, so that once the system is running the addition of a new applications becomes easy.

Another important fact that has to be emphasized is that, in contrast to traditional architectures, service oriented architectures do not force all services to access all middleware/layers available (as can be seen in figure 2). For instance, in order to check battery levels, communication protocols can be bypassed and access to physical level directly used, making signal processing more efficient.

All in all, an interoperable, reliable system, abstracting the hardware from the applications has been designed, developed and implemented. It is important to highlight that the size of the prototype is not comparable to that of wearable devices, but the aim of this work was just to emulate the behavior that wearable sensors will have in the future, not to miniaturize existing ones.

\section{CONCLUSIONS}

Deepening in the usability concept, this project has aimed to develop an intelligent and intuitive interface, embedded in everyday objects, able to respond to the activities and situations recognized in the user's environment.

The primary objective of this platform is to examine whether a system of basic biomedical sensors (motion sensors, ecg, pulse oximetry, blood pressure, etc.) is able to detect behavioral patterns and help aging and ill populations with everyday activities and health care follow up. That is to say, to provide the foundation for an Ambient Intelligence approach.

Finally, a flexible, dynamic and easy to program architecture has been implemented, enabling software developers to focus on application level without paying attention to network and hardware levels.

\section{ACKNOWLEDGMENT}

The authors would like to thank Pedro Malagón for his support during the development of the system.

\section{REFERENCES}

[1] Ades PA, Pashkow FJ, Fletcher G, Pina IL, Zohman LR, Nestor JR. A controlled trial of cardiac rehabilitation in the home setting using electrocardiographic and voice transtelephonic monitoring. Am Heart J. vol 139, sup 3, pp 543-8. 2000.

[2] Morlion B, Knoop C, Paiva M, Estenne M. Internet-based home monitoring of pulmonary function after lung transplantation. American Journal of Respiratory and Critical Care Medicine vol 165, sup 5, pp 694-697. 2002.

[3] Hernandez C, Casas A, Escarrabill et al. Home hospitalization of exacerbated COPD patients. A randomized controlled trial of clinical efficacy and costs of services for chronically ill patients. ERJ vol 21, sup 1, pp 58-67. 2003

[4] Trivedi, M.M., Huang, K.S., Mikic, I. Dynamic context capture and distributed video arrays for intelligent spaces. Systems, Man and Cybernetics, Part A, IEEE Transactions on, vol. 35, sup. 1, pp 145163, 2005 (ISSN: 1083-4427).

[5] Cucchiara, R., Grana, C., Prati, A., Vezzani, R. Probabilistic posture classification for Human-behavior analysis. Systems, Man and Cybernetics, Part A, IEEE Transactions on, vol. 35, sup. 1, pp 42-54, 2005 (ISSN: 1083-4427).

[6] Remagnino, P.; Foresti, G.L. Ambient Intelligence: A New Multidisciplinary Paradigm. Systems, Man and Cybernetics, Part A, IEEE Transactions on, vol. 35, sup. 1, pp 1-6, 2005 (ISSN: 10834427)

[7] Information Society Technologies Advisory Group. ISTAG, Scenarios for Ambient Intelligence in 2010. http://www.cordis.lu/istag.htm 2002

[8] M.Sgroi, et al. A Service-Based Universal Application Interface for Ad-hoc Wireless Sensor Networks. Junio, 2003

[9] W.B. Heinzelman, et al. Middleware to Support Sensor Network Applications. IEEE Network Magazine Special Issue. 2004

[10] A.Ranganathan, R.H.Campbell. A Middleware for Context-Aware Agents in Ubiquitous computing Environments Department of Computer Science. University of Illinois at Urbana-Champaign, USA 2003

[11] S. Coleri, A. Puri, and P. Varaiya. Power Efficient system for Sensor Networks. 8th IEEE International Symposium on Computers and Communication Proceedings (ISCC 2003). 2003

[12] J.M. Rabaey, et al. PicoRadio supports ad hoc ultra-low power wireless networking. IEEE Computer, 33, 7, pp 42-48. 2000 